\title{
Avaliação do comportamento reológica e mecânico de geopolímero sintetizado com reaproveitamento de resíduo
}

\author{
Evaluation of the rheological and mechanical \\ behavior of the synthesized geopolymer \\ with residue waste
}

Woshington da Silva Brito', Sebastião Martins Brum ${ }^{2}$, André Luis Mileo Ferraioli Silva ${ }^{2}$, Augusta Maria Paulain Ferreira Felipe ${ }^{1}$, José Antônio da Silva Souza ${ }^{2}$

\footnotetext{
${ }^{1}$ UFPA/ITEC/Departamento de Engenharia Química CEP: 66075-110, Belém, PA, Brasil. e-mail:wsbrito3@gmail.com, smartinsbrum@yahoo.com.br, ampf@ufpa.br

${ }^{2}$ UFPA/ITEC/PRODERNA - programa de Pós-Graduação em Engenharia de Recursos Naturais da Amazônia, Belém, PA, Brasil.

e-mail: adremileo@ufpa.br, jass@ufpa.br
}

\section{RESUMO}

A construção sustentável do futuro, além de ter baixo consumo de energia e emissões de gases de efeito estufa também deve adotar o princípio do reaproveitamento de resíduos impactantes ao meio ambiente gerado pela cadeia produtiva. A cadeia produtiva do alumínio tem como um dos principais resíduos impactante ao meio ambiente a geração de cinza volante. Os geopolímeros são materiais cimentícios com estrutura tridimensional formados por ativação química de aluminossilicatos. De acordo com estudos algumas cinzas vêm se mostrando adequadas como fonte de Al e Si na reação de geopolimerização. Um dos aspectos mais importantes para a comercialização desses produtos é o comportamento deles em estado plástico. A trabalhabilidade da pasta de geopolímero fresco pode ser medida com vários testes comuns usados para concreto de cimento Portland, como fluxo e queda. No entanto, uma caracterização mais aprofundada de sua reologia é essencial para entender seus mecanismos básicos de configuração. $\mathrm{O}$ trabalho de pesquisa tem como objetivo avaliar o comportamento reológico da pasta de geopolimero com razão de Davidovits $\left(\mathrm{SiO}_{2} / \mathrm{Al}_{2} \mathrm{O}_{3}\right)$ de 2,65; 3,04 e 4,11. Foi avaliado também a resistência mecânica a compressão com 24 horas, 7 dias e 28 dias de cura de geopolímero. A reação de geopolimerização foi conduzida a temperatura ambiente de $28^{\circ} \mathrm{C}$ e como ativador utilizou-se hidróxido de sódio $(\mathrm{NaOH}) 15$ Molar e silicato de sódio $\left(\mathrm{Na}_{2} \mathrm{SiO}_{3}\right)$ alcalino 10 Molar. Como fonte de $\mathrm{Al}$ e $\mathrm{Si}$ utilizou-se cinza volante e metacaulim. Técnicas de DRX, FRX, MEV foram utilizadas na caracterização das matérias primas e dos geopolímeros. O geopolímero com menor razão de Davidovits 2,65 apresentou melhor trabalhabilidade no seu estado fresco e maior resistência mecânica a compressão de 40,80 MPa com 28 dias de cura a temperatura ambiente. O modelo reológico Herchel Bulkley foi o que melhor se ajustou aos geopolímeros.

Palavras-Chave: geopolímero; reologia; modelo reológico

\begin{abstract}
The sustainable construction of the future, besides low energy consumption and greenhouse gas emissions must also adopt the principle of reusing wastes generated by the production chain that impact the environment. One of the most impacting wastes generated by the aluminum production chain is fly ash. Geopolymers are cementitious materials with a three-dimensional structure formed by chemical activation of aluminosilicates. According to studies some ashes have proven to be suitable sources of $\mathrm{Al}$ and $\mathrm{Si}$ for the geopolymerization reaction. One of the most important aspects in the commercializing these products is their behavior in a plastic state. The workability of fresh geopolymer paste can be measured using several common tests used for Portland cement concrete, such as flow and slump; however, a more in-depth characterization of their rheology is essential for understanding their
\end{abstract}


basic setting mechanisms. The objective of this work was to evaluate the rheological behavior of the Davidovits $\left(\mathrm{SiO}_{2} / \mathrm{Al}_{2} \mathrm{O}_{3}\right)$ geopolymer paste at $2.65 ; 3.04$ and 4.11 . The mechanical resistance to compression at 24 hours, 7 days and 28 days of geopolymer cure was also evaluated. The geopolymerization reaction was conducted at ambient temperature of $28^{\circ} \mathrm{C}$ and 15 Molar sodium hydroxide $(\mathrm{NaOH})$ and 10 Molar sodium silicate $(\mathrm{Na} 2 \mathrm{SiO} 3)$, were used as the activator. Fly ash and metakaolin were used as source of $\mathrm{Al}$ and $\mathrm{Si}$ was used. XRD, XRF, SEM techniques were used in the characterization of raw materials and geopolymers. The geopolymer with a lower Davidovits ratio of 2.65 presented better workability in its fresh state and higher mechanical strength at $40.80 \mathrm{MPa}$ compression with 28 days cure at ambient temperature. The Herchel Bulkley rheological model was the one that was best adjusted to the geopolymers.

Keywords: geopolymer; rheology; rheological model

\section{INTRODUÇÃO}

Os geopolímeros são sintetizados a diferentes temperaturas pela ativação alcalina de aluminossilicatos derivados de minerais naturais, argila calcinada ou subprodutos industriais 2008. Geralmente, esta ativação é realizada com metacaulim silicatos de sódio ou de potássio. Os geopolímeros são ligantes inorgânicos com boa resistência a alta temperatura e a degradação de ácido, bem como boas propriedades mecânicas. Portanto, é uma alternativa atraente ao cimento do tipo Portland, e seu uso permite a reciclagem de grandes quantidades de resíduos industriais. As propriedades mecânicas dos materiais geopoliméricos dependem do cátion alcalino $\left(\mathrm{Na}^{+}\right)$, da relação molar $\mathrm{SiO}_{2} / \mathrm{Al}_{2} \mathrm{O}_{3}$ conforme utilizado por Davidovits e das condições com que a reação ocorre [9], [14].

O termo "geopolímero" descreve as propriedades químicas dos polímeros inorgânicos à base de aluminossilicatos. Os geopolímeros apresentam propriedades de cimento e, portanto, um grande potencial de uso na indústria da construção civil [4].

A reologia dos geopolímeros no estágio inicial antes da configuração endurecida é de grande importância no entendimento do comportamento da pasta no estado plástico e avaliar sua aplicação [12].

Medidas reológicas são usadas como um método indireto no entendimento e monitoramento da reação de geopolimerização em seu período inicial [11].

Alterar a relação $\mathrm{SiO}_{2} / \mathrm{Al}_{2} \mathrm{O}_{3}$ em geopolímeros permite assim a síntese de materiais com diferentes estruturas. $\mathrm{O}$ mecanismo de geopolimerização é particularmente difícil devido à cinética da reação. No entanto, a maioria dos autores concorda que o mecanismo envolve a dissolução, seguido de policondensação de gel [7].

Estudos mostram que as forças mecânicas dos geopolímeros aumentam com a elevação da temperatura de calcinação do caulim para gerar metacaulim, no entanto, a temperatura ideal para a calcinação do caulim é de cerca de $700{ }^{\circ} \mathrm{C}$ por 2 horas [16], [3].

O desenvolvimento de geopolímero a base de cinza proveniente da queima de carvão vem se mostrando promissor pelo reaproveitamento desse resíduo. A demanda global por carvão crescerá até 2030, atingindo uma duplicação em relação à demanda atual. Com o crescente consumo de carvão para a geração de energia, haverá uma produção aumentada de cinzas como um subproduto da combustão do carvão [2].

\section{MATERIAIS E MÉTODOS}

O resíduo cinza volante foi coletado no overflow do ciclone das caldeiras de leito fluidizado tipo circulante em condições de $900^{\circ} \mathrm{C}$ de temperatura e $120 \mathrm{KPa}$ de pressão. Esse resíduo foi utilizado como fonte de $\mathrm{Si}$ e $\mathrm{Al}$ na síntese de geopolímero. Para aumentar a reatividade da reação de geopolimerização foi utilizado também como fonte de $\mathrm{Si}$ e $\mathrm{Al}$ o metacaulim produzido pela calcinação de caulim em temperatura de $800^{\circ} \mathrm{C}$ por duas horas.

Para as análises da estrutura cristalina da cinza, do caulim e do metacaulim foi utilizado difratômetro de raios X (DRX) de marca Bruker LyuxEye. Já para análises químicas usou-se fluorescência de raios X (FRX) em um equipamento da marca AxiosMinerals (PANalytical), com tubo de raios- $X$ cerâmico e anodo de ródio. A Cinza e o metacaulim foram submetidos a analise granulométrica de suas partículas, em granulômetro a laser de marca Fritsch Analysette 22 Micro tec plus. 
Foi formulado 3 composições de geopolímeros a base de cinza volante e metacaulim com diferentes razões molares entre $\mathrm{SiO}_{2} / \mathrm{Al}_{2} \mathrm{O}_{3}$. A Tabela 1 apresenta os geopolímeros estudados com suas respectivas razões de $\mathrm{SiO}_{2} / \mathrm{Al}_{2} \mathrm{O}_{3}$.

Tabela 1: Composições formuladas entre as cinzas volante e o metacaulim para obtenção dos geopolímeros.

\begin{tabular}{cccc}
\hline Geopolímeros & Cinza volante (\%) & Metacaulim $(\%)$ & $\mathbf{S i O}_{\mathbf{2}} / \mathbf{A l}_{\mathbf{2}} \mathbf{O}_{\mathbf{3}}$ \\
\hline \hline $\mathrm{A}$ & 94,24 & 5,76 & 4,11 \\
$\mathrm{~B}$ & 61,54 & 38,46 & 3,04 \\
$\mathrm{C}$ & 42,31 & 57,69 & 2,65 \\
\hline
\end{tabular}

$\%$ - Em massa

As pastas dos geopolímeros A, B e C no estado fresco foram submetidos a ensaio reológico a temperatura de $28^{\circ} \mathrm{C}$ em viscosímetro modelo Haake de cilindros coaxiais tipo SV1.

O ativador alcalino usado na síntese do geopolímero foi composto de uma solução de hidróxido de sódio $(\mathrm{NaOH})$ micro perola (Neon, $97 \%$ pureza). Também, usou-se solução de silicato de sódio alcalino $\left(\mathrm{Na}_{2} \mathrm{SiO}_{3}\right)$ (Manchester Química do Brasil S.A., $\left.\mathrm{SiO}_{2} / \mathrm{Na}_{2} \mathrm{O}=3,20\right)$. A Tabela 2 apresenta a composição do ativador:

Tabela 2: Concentração molar e proporções da solução ativadora

\begin{tabular}{cc|c}
\hline \hline Ativador: & $\mathrm{NaOH}$ & $\mathrm{Na}_{2} \mathrm{SiO}_{3}$ \\
\cline { 2 - 3 } Concentração Molar: & 15 & 10 \\
\cline { 2 - 3 } Proporção $\left(\mathbf{N a O H}: \mathbf{N a}_{2} \mathbf{S i O}_{3}\right):$ & $1: 3$ em massa de solução \\
\hline \hline
\end{tabular}

As composições foram preparadas em misturador mecânico e conformadas em moldes cilíndricos de $100 \mathrm{~mm}$ de altura e diâmetro de $50 \mathrm{~mm}$. Após a moldagem os moldes foram submetidos a cura a temperatura ambiente de $28^{\circ} \mathrm{C}$. Os geopolímeros com 24 horas, 7 e 28 dias de cura foram submetidos a ensaios de resistência a compressão em prensa Emic SSII300.

O geopolímero $\mathrm{C}$ após sofrer ensaio de resistência a compressão foi submetido a ensaio de EDS em equipamento Zeiss MEO 1430 como forma de avaliar sua composição e morfologia no estado endurecido.

\section{RESULTADOS E DISCUSSÃO}

A tabela 3 apresenta os resultados das análises de FRX dos materiais utilizados na síntese do geopolímero. Observa-se (tabela 3) o teor de 42,53\% de $\mathrm{SiO}_{2}$ e $16,39 \%$ de $\mathrm{Al}_{2} \mathrm{O}_{3}$ na cinza volante usada, o que indica que a cinza é uma possivel fonte de Al e Si. Para aumentar as propriedades reativas da cinza foi adicionado metacaulim (tabela 3 ) é composto por 43,58\% de $\mathrm{Al}_{2} \mathrm{O}_{3}$ e $53,36 \% \mathrm{SiO}_{2}$, o que caracteriza como fonte predominante de alumínio em proporção [15], [17], [6], [1].

Tabela 3: Composição química em \% da cinza e do metacaulim.

\begin{tabular}{cccccccccc}
\hline Material & $\mathbf{S i O}_{\mathbf{2}}$ & $\mathbf{A l}_{\mathbf{2}} \mathbf{O}_{\mathbf{3}}$ & $\mathbf{C a O}$ & $\mathbf{F e}_{\mathbf{2}} \mathbf{O}_{\mathbf{3}}$ & $\mathbf{N a}_{\mathbf{2}} \mathbf{O}$ & $\mathbf{M g O}$ & $\mathbf{T i O}_{\mathbf{2}}$ & $\mathbf{P}_{\mathbf{2}} \mathbf{O}_{\mathbf{5}}$ & $\mathrm{Loi}$ \\
\hline \hline CINZA & 42,53 & 16,39 & 19,05 & 7,08 & 0,94 & 0,26 & 0,89 & 0 & 12,86 \\
METACAULIM & 53,36 & 43,58 & 0 & 0,60 & 0,33 & 0 & 1,51 & 0,13 & 0,49 \\
\hline \hline
\end{tabular}

Pelos resultados das composições químicas obtidos na tabela 3 , confirma-se as razões de $\mathrm{SiO}_{2} / \mathrm{Al}_{2} \mathrm{O}_{3}$ nos valores de 4,11; 3,04 e 2,65 para os geopolímeros $\mathrm{A}$, B e C respectivamente.

A Figura 1 apresenta o resultado do ensaio do DRX da cinza volante, do caulim e do metacaulim 
calcinado a $800{ }^{\circ} \mathrm{C}$ por 2 horas.
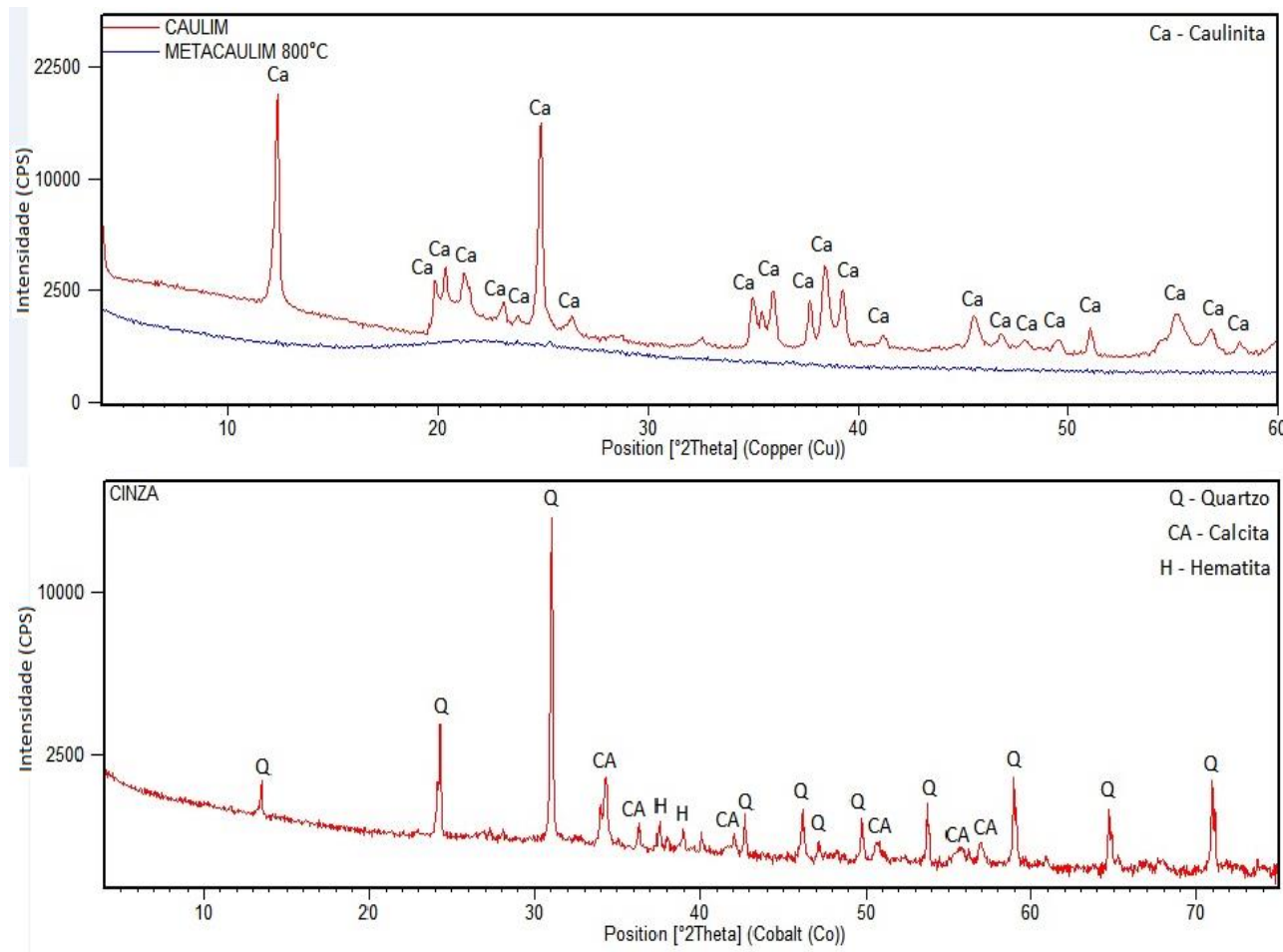

Figura 1: DRX do caulim, do metacaulim e da cinza volante.

Conforme demonstrado na Figura 1, a calcinação do caulim a $800^{\circ} \mathrm{C}$ por duas horas foi satisfatória, pois gerou uma mudança na fase cristalina transformando-a em fase amorfa, caracterizando o metacaulim. A mudança ocorrida no material após o tratamento térmico libera $\mathrm{Al}$ e o Si para reação de geopolimerização. Caulim calcinado numa faixa entre $600^{\circ} \mathrm{C}$ e $800^{\circ} \mathrm{C}$ por um intervalo de tempo entre $2 \mathrm{e}$ 4 horas, vem se mostrando adequado na obtenção de metacaulim aplicado ao desenvolvimento de geopolímero [5], [10].

A cinza volante gerada nas refinarias de alumínio apresenta quartzo e calcita em sua constituição como evidenciado no difratograma da cinza na Figura 1. A distribuição de tamanho de partícula das materiais primas usadas na síntese do geopolímero resultou em um diâmetro médio $\left(\mathrm{d}_{50}\right)$ de $24 \mu \mathrm{m}$ para a cinza volante e de $88 \mu \mathrm{m}$ para o metacaulim.

A figura 2 apresenta os resultados de resistência mecânica a compressão em MPa, indicando que o geopolímero C possui maior resistência mecânica em comparação ao geopolímeros B e A.

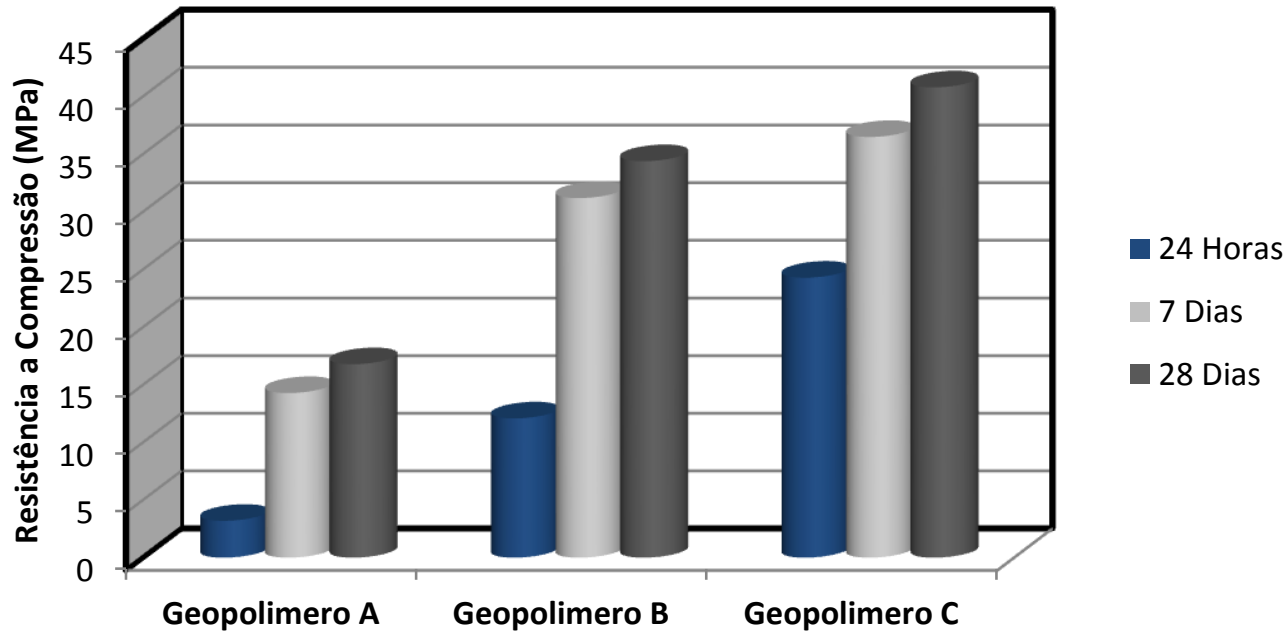


Figura 2: Resistência mecânica a compressão

$\mathrm{O}$ geopolímero $\mathrm{C}$ com razão de $2,65\left(\mathrm{SiO}_{2} / \mathrm{Al}_{2} \mathrm{O}_{3}\right)$ apresentou alta resistência a compressão atingindo valores de 24,31 $\mathrm{MPa}, 36,53 \mathrm{Mpa}$ e 40,80 $\mathrm{MPa}$ em 24 horas, 7 dias e 28 dias de cura, respectivamente a temperatura ambiente. Pela Figura 2 o geopolímero $\mathrm{C}$ atingiu em 24 horas a resistência de concreto do tipo Portland convencional com 28 dias de cura.

A figura 3 apresenta as curvas de viscosidade aparente e de tensão versus taxa de cisalhamento, em que os modelos reológicos foram ajustados, para os geopolímeros A, B e C.

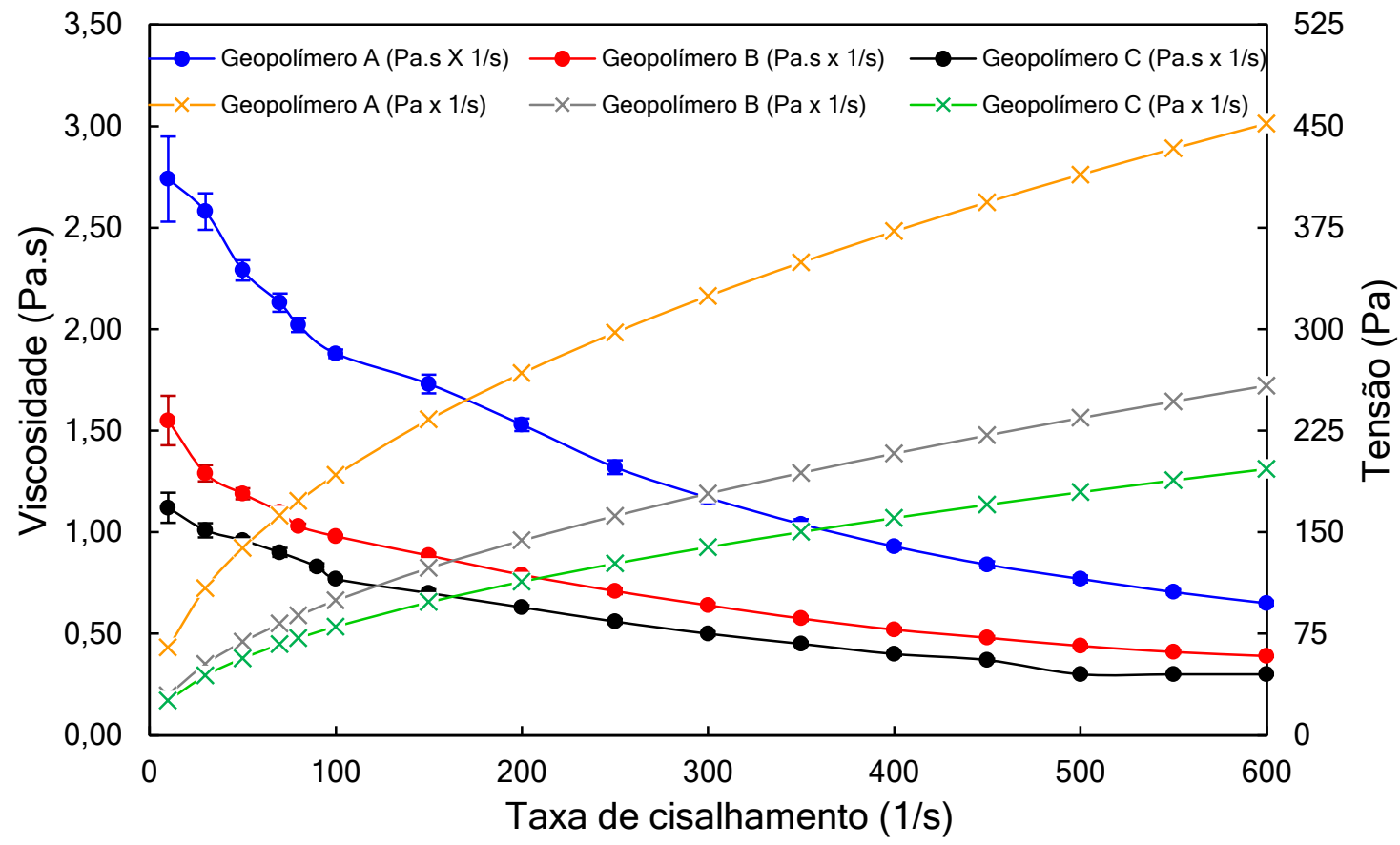

Figura 3: Curvas de viscosidade aparente e tensão versus taxa de cisalhamento

De acordo com a figura 3 o aumento da quantidade de resíduo cinza volante na composição do geopolímero, contribui com o aumento da viscosidade aparente, conferindo ao geopolímero uma diminuição na trabalhabilidade da pasta. O aumento da incorporação de cinza volante na pasta geopolimérica contribui com a elevação da viscosidade e da tensão devido ao aumento de finos na composição.

Pela figura 2 e 3 o geopolímero com razão de 2,65 $\left(\mathrm{SiO}_{2} / \mathrm{Al}_{2} \mathrm{O}_{3}\right)$, geopolímero $\mathrm{C}$, além de apresentar maior resistência mecânica a compressão possui também melhor trabalhabilidade devido sua menor resistência ao escoamento, ou seja, menor viscosidade aparente $(\boldsymbol{\eta})$.

A tabela 4, 5 e 6 apresentam os modelos reológicos, com seus respectivos parâmetros e equações, que representam o comportamento de escoamento do geopolímero A, B e C respectivamente.

Tabela 4: Modelo Reológico para o geopolímero A

\begin{tabular}{ccccc}
\hline Geopolímero A & $\tau_{\circ}$ & $\mathbf{K}$ & $\mathbf{N}$ & $\mathbf{R}^{2}$ \\
Herschel-Bulkley & & & & \\
$\eta=\frac{\tau_{\circ}}{\dot{\gamma}}+\kappa \gamma^{n-1}$ & 1,92 & 20,85 & 0,4802 & 0,9926 \\
Geopolímero A & & & & \\
Ostwald de Waele & - & $\mathbf{K}$ & $\mathbf{N}$ & $\mathbf{R}^{\mathbf{2}}$ \\
\hline
\end{tabular}




$$
\eta=k \cdot \gamma^{n-1}
$$

\begin{tabular}{ccccc}
\hline $\begin{array}{c}\text { Geopolímero A } \\
\text { Bingham }\end{array}$ & $\tau_{\circ}$ & $\eta_{p}$ & - & $\mathbf{R}^{2}$ \\
$\eta=\eta_{p}+\frac{\tau_{\circ}}{\dot{\gamma}}$ & 90,34 & 0,7545 & & 0,9771 \\
\hline
\end{tabular}

Tabela 5: Modelo Reológico para o geopolímero B

\begin{tabular}{ccccc}
\hline $\begin{array}{c}\text { Geopolímero B } \\
\text { Herschel-Bulkley }\end{array}$ & $\tau_{\circ}$ & $\mathbf{K}$ & $\mathbf{N}$ & $\mathbf{R}^{2}$ \\
$\begin{array}{c}\eta=\frac{\tau_{\circ}}{\dot{\gamma}}+\kappa \gamma^{n-1} \\
\text { Geopolímero B }\end{array}$ & 0,69 & 8,42 & 0,5347 & 0,9942 \\
$\begin{array}{c}\text { Ostwald de Waele } \\
\eta=k . \gamma^{n-1}\end{array}$ & - & $\mathbf{K}$ & $\mathbf{N}$ & $\mathbf{R}^{2}$ \\
\hline $\begin{array}{c}\text { Geopolímero B } \\
\text { Bingham }\end{array}$ & $\tau_{\circ}$ & $\eta_{p}$ & 0,6309 & 0,9919 \\
$\eta=\eta_{p}+\frac{\tau_{0}}{\dot{\gamma}}$ & 41,55 & 0,4428 & & $\mathbf{R}^{2}$ \\
\hline
\end{tabular}

Tabela 6: Modelo Reológico para o geopolímero C

\begin{tabular}{ccccc}
\hline $\begin{array}{c}\text { Geopolímero C } \\
\text { Herschel-Bulkley }\end{array}$ & $\tau_{\circ}$ & $\mathbf{K}$ & $\mathbf{N}$ & $\mathbf{R}^{2}$ \\
$\begin{array}{c}\eta=\frac{\tau_{\circ}}{\dot{\gamma}}+\kappa \gamma^{n-1} \\
\text { Geopolímero C }\end{array}$ & 0,53 & 7,87 & 0,5028 & 0,9949 \\
Ostwald de Waele & - & $\mathbf{K}$ & $\mathbf{N}$ & $\mathbf{R}^{2}$ \\
$\eta=k \cdot \gamma^{n-1}$ & - & 8,05 & 0,4994 & 0,9937 \\
\hline Geopolímero C & $\tau_{\circ}$ & $\eta_{p}$ & - & $\mathbf{R}^{2}$ \\
Bingham & & 0,3330 & & 0,9780 \\
$\eta=\eta_{p}+\frac{\tau_{0}}{\dot{\gamma}}$ & 35,84 & & & \\
\hline
\end{tabular}




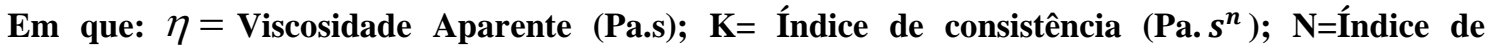
comportamento (adimensional); $\dot{\gamma}=$ Taxa de deformação $\left(\mathrm{s}^{-1}\right) ; \eta_{p}=$ Viscosidade plástica; $\tau_{\circ}=$ Tensão limite de escoamento.

Pelos resultados das tabelas 4, 5 e 6, observa-se entre os modelos de Herschel-Bulkley, Ostwald de Waele e Binghan o que melhor se ajustou ao comportamento reológico da pasta fresca tanto do geopolímero C quanto dos geopolímeros A e B foi o modelo de Herschel-Bulkley, devido seu maior coeficiente de determinação $\mathrm{R}^{2}$. O maior coeficiente de determinação é o fator determinante, pois, indica o conjunto numérico de parâmetros $\left(\mathrm{N}, \mathrm{K}, \eta_{p}\right.$ e $\left.\tau_{\circ}\right)$ do modelo reológico que melhor se ajustou aos dados experimentais.

De acordo com as tabelas 4,5 e 6 os geopolímeros A, B e C apresentam comportamento não newtoniano, pois, a viscosidade aparente $(\boldsymbol{\eta})$ decresce com o aumento da taxa de cisalhamento $(\dot{\gamma})$ e o índice de comportamento $(\mathrm{N})$ de todos os geopolímeros foram menor que 1.

O modelo de Ostwald-de-Waele, assim como o de Herschel- Bulkley, considera a relação não linear entre a tensão e a taxa. Porém, o modelo de Ostwald- de- Waele não leva em consideração a tensão limite de escoamento $\left(\tau_{\circ}\right)$.

A figura 4 apresenta o EDS da estrutura do geopolímero C após sofrer o ensaio de resistência a compressão.

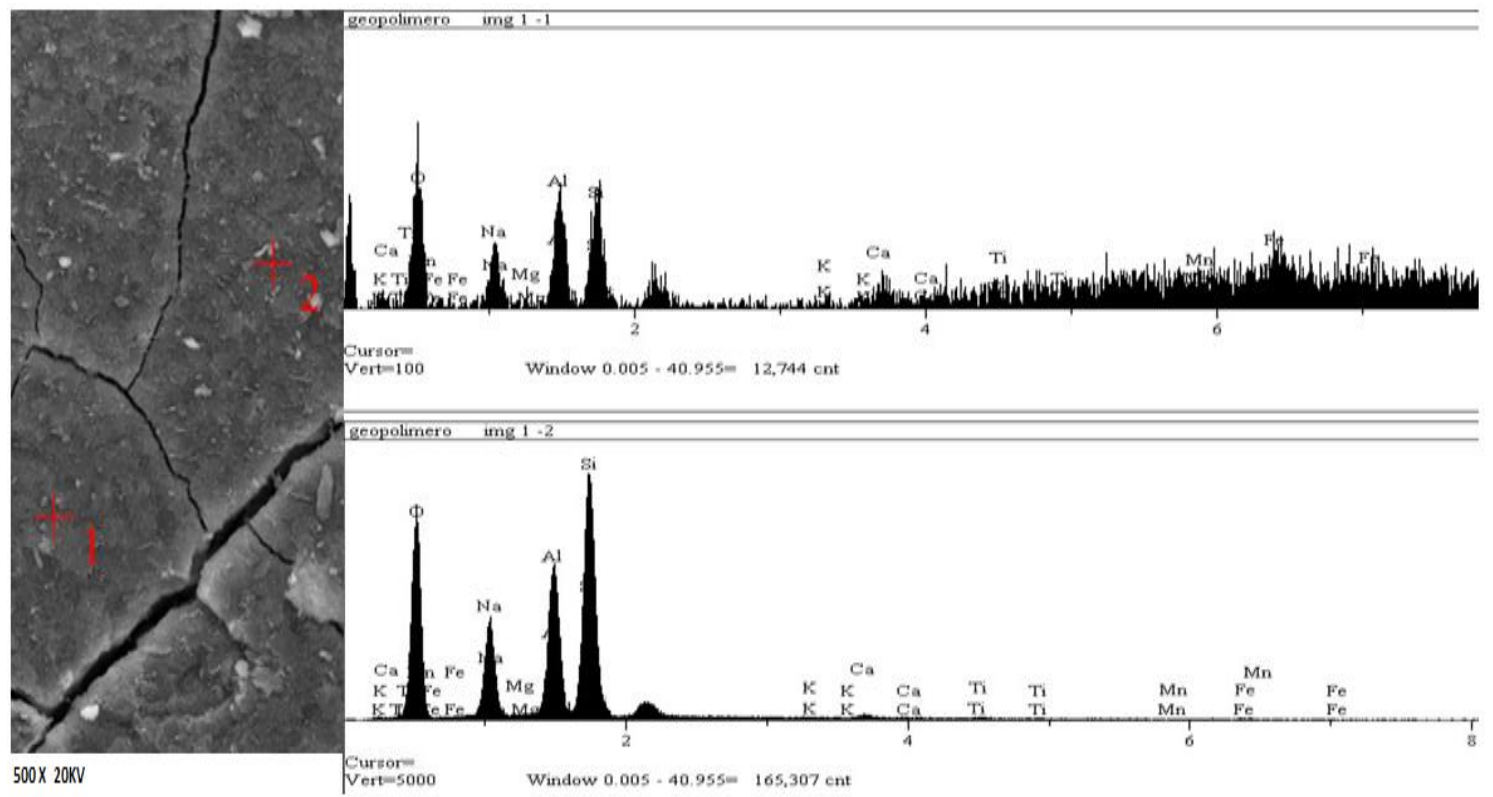

Figura 4: EDS do Geopolimero C

Pela figura 4 observa-se uma morfologia densa e uniforme do geopolímero $\mathrm{C}$ o que lhe confere alta resistência a compressão. Salehi [13] constatou em seus estudos que o aspecto morfológico mais denso e uniforme confere maior desempenho mecânico aos geopolímeros e consequentemente maior avanço de reação de geopolimerização.

No espectro do EDS da figura 4 a presença do Al e do Si enfatiza o caráter alumínio silicato da reação de geopolimerização. A presença do Na no espectro é devido ao ativador a base de hidróxido de sódio.

\section{CONCLUSÃO}

Pela pesquisa conclui-se que o geopolimero $\mathrm{C}$ a base de metacaulim e cinza volante apresentou maior grau de reação de geopolimerização indicado pela maior resistência a compressão obtida e apresentando também melhor trabalhabilidade que os geopolimeros A e B. Todos os geopolímeros A, B e C possuem pasta fresca com comportamento reológico ajustável ao modelo de Herschel-Bulkley com coeficiente de determinação superior a 0,9925 .Os geopolímeros sintetizados a temperatura ambiente com ativador em composição de 15 molar de hidróxido de sódio e 10 molar de silicato de sódio alcalino apresentaram-se 
como uma alternativa para suprir a demanda na área cimenticia, atingindo resistência superior a resistência de concretos convencionais a base de cimento portland. Além disso, os geopolímeros foram produzidos com baixa emissão de $\mathrm{CO}_{2}$, quando comparado aos cimentos convencionais, o que contribui com o meio ambiente. A pesquisa conclui que os geopolímeros sintetizados possuem grande potencial para a produção de materiais geopolimericos.

\section{BIBLIOGRAFIA}

[1] ARDANUY, M. CLARAMUNT, J. FILHO, R. D. T. "Cellulosic fiber reinforced cement-based composites: a review of recent research", Constr. Build. Mater., v .79, 115-128, 2015

[2] CASTRO-GOMES, J.P., SILVA, A.P., CANO, R.P., DURÁN SUAREZ, J., et al. "Potential for reuse of tungsten mining waste-rock in technical-artistic value added products", J. Clean. Prod. V. 25, pp 34 41. 2012

[3] CIOFFI, R., MAFFUCCI, L., SANTORO, L. "Optimization of geopolymer synthesis by calcination and polycondensation of a kaolinitic residue”, Resour Conserv Recy, v.40, pp. 27-38. 2003.

[4] DAVIDOVITS, J. Geopolymer Chemistry and Applications, third ed. Institut Géopolymère, SaintQuentin, France, 2011

[5] DAVIDOVITS, J. Geopolymers based on natural and synthetic metakaolin - A critical review, Elsevier-Geopolymer Institute Virtual Special Issues. 2015.

[6] DUAN, P., YAN, C.J., LUO, W.J., et al., "A novel surface waterproof geopolymer derived from metakaolin by hydrophobic modification”, Mater. Lett. V .164, pp .172-175, 2016

[7] DUXSON, P., FERNÁNDEZ-JIMÉNEZ, A., PROVIS, J. L., et al., "Geopolymer technology: the current state of the art", J. Mater. Sci., pp.2917-2933, 2007

[8] DUXSON, P., FERNANDEZ, J.A., PROVIS, J.L., et al., "The role of inorganic polymer technology in the development of 'green concrete' ", Mater. Sci., v. 42, pp 2917-2933, 2007.

[9] DUXSON, P., PROVIS, J.L., LUKEY, G.C., et al., "Understanding the relationship between geopolymer composition, microstructure and mechanical properties", Colloids and Surfaces A: Physicochemical and Engineering Aspects. v. 269, pp 47-58, 2005.

[10] MEDRI, V., FABBRI, S., DEDECEK, J., et al., "Role of the morphology and the dehydroxylation of metakaolins on geopolymerization”, Appl. Clay Sci., v. 50, pp .538-545, 2010.

[11] PALOMO A, BANFILL PFG, FERNÁNDEZ-JIMÉNEZ A, Swift DS. "Properties of alkaliactivated fly ashes determined from rheological measurements", Adv Cem Res., v .17, pp. 143-51, 2005.

[12] RAHIER H, VAN MELE B, WASTIELS J. "Low-temperature synthesized aluminosilicate glasses: Part II. Rheological transformations during lowtemperature cure and high-temperature properties of a model compound", J Mater Sci . v.31, pp. 80-85, 1996.

[13] SALEHI, S., KHATTAK, M. J., BWALA, A. H., et al., "Characterization, morphology and shear bond strength analysis of geopolymers: Implications for oil and gas well cementing applications", Journal of Natural Gas Science and Engineering, v. 38, pp .323-332. 2017

[14] SALIH, M. A, ABANG, A. A. A, FARZADNIA N. "Characterization of mechanical and microstructural properties of palm oil fuel ash geopolymer cement paste". Constr Build Mater. V.65, pp 592-603, 2014.

[15] TCHAKOUTE, H. K., RÜSCHER, C. H., DJOBO, J.N.Y., et al., "Influence of gibbsite and quartz in kaolin on the properties of metakaolin-based geopolymer cements", Appl. Clay Sci. V. 107, pp .188-194, 2015.

[16] WANG, M.R., JIA D.C, HE, P.G., "Influence of calcination temperature of kaolin on the structure and properties of final geopolymer”, Materials Letters. v.64, 2551-2554, 2010

[17] ZHANG, H.Y., KODUR, V., QI, S.L., et al., "Development of metakaolin-fly ash based geopolymers for fire resistance applications", Constr. Build. Mater. v. 55, pp .38-45, 2014 